\title{
Singularities of Scattering Amplitudes on Unphysical Sheets and Their Interpretation
}

\author{
R. Blankenbecler, M. L. Goldberger, S. W. MacDowell,* and S. B. Treiman \\ Palmer Physical Laboratory, Princeton University, Princeton, New Jersey $\dagger$
}

(Received March 1, 1961)

\begin{abstract}
The analytic structure of two-particle scattering amplitudes on the unphysical sheet of the Riemann surface reached by crossing the two-particle cut is discussed. The singularities of the amplitudes there are shown to be poles and their physical interpretation is studied. The way in which bound states appear on the physical sheet in the Mandelstam representation, both as isolated poles and as cuts, is traced in detail. The properties of partial wave amplitudes and of the full amplitude as a function of energy and angle and of energy and momentum transfer are discussed. Finally, a few remarks are made in connection with unstable states.
\end{abstract}

\section{INTRODUCTION}

A NY systematic program to explore the analytic structure of a scattering amplitude which restricts itself to the physical Riemann sheet in the energy variables does not seem complete. The properties on the physical sheet must be supplemented by a knowledge of the analytic behavior on the second Riemann sheet as well as some idea of the dependence of the position of any singularities on the parameters of the theory. For example, the simplest way in which the analytic properties on the physical sheet can change as some parameter of the theory is varied is that singularities on the second sheet migrate to the physical sheet through branch cuts already present.

Such a behavior occurs in the problem of anomalous thresholds. ${ }^{1,2}$ It is found that as the external masses increase, a branch cut moves through the normal cut onto the physical sheet and extends the threshold below the canonical value. As is demonstrated later, the formation of bound states in field theory is also a matter of poles and cuts moving to the physical sheet as the interaction becomes more and more attractive.

The problem of unstable particles and the resulting scattering resonances has also been discussed in terms of poles on the second Riemann sheet. The conjecture of Peierls ${ }^{3}$ is that a pole on the second sheet is to be identified with an unstable particle. These poles depended strongly on details of the theory and their physical interpretation is not clear. It is not clear to us, for example, what characteristics the singularities on the unphysical sheet defined by crossing the threeparticle branch cut must have in order to yield a consistent physical interpretation. These singularities are discussed, and are shown to have a reasonable interpretation in terms of an unstable particle. Due to

\footnotetext{
* Present address: Centro Brasileiro de Pesquisas, Fisicas, av Venceslau Braz. 71, Estado da Guanabara, Brasil.

$\dagger$ Supported in part by the Air Force Office of Scientific Research, Air Research and Development Command.

${ }^{1}$ S. Mandelstam, Phys. Rev. Letters 4, 84 (1960).

2 R. Blankenbecler and Y. Nambu, Nuovo cimento 18, 595 (1960)

${ }_{3}^{3}$ R. E. Peierls, Proceedings of the 1954 Glasgow Conference on Nuclear and Meson Physics (Pergamon Press, New York, 1954), p. 296. See also J. Gunson and J. G. Taylor, Phys. Rev. 119, 1121 (1960).
}

our lack of knowledge of unitarity, we are unable to make very definite statements in the inelastic case. However, we prove that in the two-particle case, the only additional singularities allowed on the unphysical sheet are poles.

It is possible to discuss the many-channel problem by utilizing the matrix formulation of Bjorken $^{4}$ and Nauenberg. ${ }^{5}$ Only the one-channel problem is discussed here, but most of the equations we develop are true in the many-channel case if they are looked upon as matrix equations.

We consider first the case of individual partial waves because the application of unitarity is so simple in this case. We further restrict our attention to the scattering of scalar "nucleons" of mass $M$, exchanging pions of mass $\mu$. It is a "simple" matter to extend the discussion to more interesting cases.

In order to discuss the convergence of a partial wave expansion on the second sheet, the full amplitude at fixed angle must be considered. This is done by assuming that a double dispersion relation holds on the physical sheet and discussing the amplitude on the second sheet by means of a Fredholm solution to the defining integral equation. Finally, the full amplitude at fixed momentum transfer is discussed.

The possibility that singularities originally on the second sheet could produce singularities not found in perturbation theory is discussed in the case of anomalous thresholds in form factors and scattering amplitudes. The problem of bound states is clarified by showing in detail how the poles and cuts associated with this mass state are produced on the physical sheet as the interaction becomes sufficiently attractive.

\section{PARTIAL WAVES}

The analytic properties of the partial wave amplitudes have been well discussed. ${ }^{6}$ The essential result is that the function defined by

$$
f_{l} \mathrm{I}(\nu+i \epsilon)=\exp [i \delta(\nu)] \sin \delta(\nu) / \rho(\nu),
$$

${ }^{4}$ J. Bjorken, Phys. Rev. Letters 4, 473 (1960).

${ }^{5} \mathrm{M}$. Nauenberg (unpublished thesis and to be published).

${ }^{6}$ S. N. MacDowell, Phys. Rev. 116, 774 (1959). 
where $\rho(\nu)=\left[\nu /\left(\nu+M^{2}\right)\right]^{\frac{1}{2}}$ is an analytic function of the square of the relative center-of-mass momentum, $\nu$, with a cut along the entire positive real axis and along the negative real axis from minus infinity to $\left(-\mu^{2} / 4\right)$ where $1 / \mu$ is the range of the effective potential. The superscript one is to emphasize that this equation is defined on the physical sheet.

Below the onset of inelastic channels, the phase shift is real along the physical cut and the unitarity relation takes the usual form. We next remark that by trivial manipulation

where

$$
f_{l}^{\mathrm{I}}(\nu-i \epsilon)=f_{l}^{\mathrm{I}}(\nu+i \epsilon) S_{l}^{-1}(\nu+i \epsilon)
$$

$$
S_{l}(\nu+i \epsilon)=\exp \left[2 i \delta_{l}(\nu)\right]=1+2 i \rho(\nu) f_{l}^{\mathrm{I}}(\nu+i \epsilon) .
$$

The scattering amplitude on the second sheet is introduced as the continuation across the positive branch cut below the inelastic threshold in a counterclockwise direction:

$$
f_{l}^{\mathrm{II}}(\nu+i \epsilon)=f_{l}^{\mathrm{I}}(\nu-i \epsilon)=f_{l}^{\mathrm{I}}(\nu+i \epsilon) S_{l}^{\mathrm{I}}(\nu+i \epsilon) .
$$

It is immediately obvious that $f_{l}^{\mathrm{II}}(\nu)$ has the same region of analyticity as $f_{l} \mathrm{I}(\nu)$ except that there may be poles due to zeroes of the $S$ matrix and the trivial kinematic cut coming from the factor of $\rho(\nu)$.

The $l$ th partial cross section, which is defined as

$$
\sigma_{l}(\nu)=f_{l}^{\mathrm{II}}(\nu) f_{l}^{\mathrm{I}}(\nu)
$$

is easily seen to enjoy analyticity in the $\nu$ plane cut along the negative axis to $\left(-\mu^{2} / 4\right)$ with poles due to the zeroes of $S_{l}$. The fact that $\sigma_{l}$ has no positive cut in the elastic region is easily demonstrated. Similar statements hold for the functions $\rho \operatorname{Im} f_{l}$ and $\operatorname{Re} f_{l}$. An interesting and amusing fact, which can be demonstrated readily by writing

is that

$$
\operatorname{Re} f_{l}^{\mathrm{I}}=f_{l}^{\mathrm{I}} S_{l}^{-1}\left[1+i \rho f_{l} \mathrm{I}\right]
$$

$$
\operatorname{Re} f_{l}^{\mathrm{I}} \rightarrow \frac{1}{2} f_{l}^{\mathrm{I}}
$$

wherever $f_{l}^{\mathrm{I}}$ approaches infinity.

Let us now see whether or not $S_{l}$ has zeroes close to the physical region. The simplest place to look for a zero is in the gap between the positive and negative cut where $S_{l}$ is real. The complex zeroes of the $S$ matrix which might lead to scattering resonances are strongly dependent upon the details of the theory and are therefore difficult to discuss in general. It can be shown that $S_{l}$ has at least one zero between $\nu=0$ and $\left(-\mu^{2} / 4\right)$ for every other $l$ if there is a one-particle exchange contribution to the negative cut and no bound states.

Since there are no bound states present, $S_{l}$ is bounded in the gap. Further, if there is no zero-energy resonance or anomalous threshold, the $S$ matrix is unity at zero kinetic energy. As $\nu$ approaches $\left(-\mu^{2} / 4\right)$, the singular
FIG. 1. $S$ matrix with no bound state.

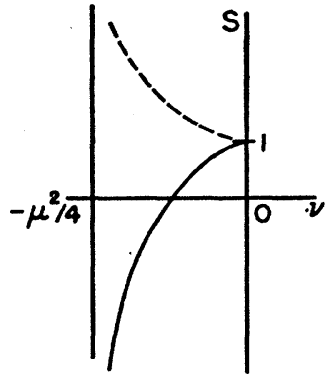

part of the Yukawa-type Born term approaches

$$
\begin{aligned}
f_{l}^{\mathrm{I}}(\nu) & \simeq-\frac{\lambda}{2 \nu} P_{l}\left(1+\frac{\mu^{2}}{2 \nu}\right) \ln \left(1+\frac{4 \nu}{\mu^{2}}\right) \\
& \simeq \frac{2 \lambda}{\mu^{2}} \ln (0+) P_{l}(-1),
\end{aligned}
$$

where $\lambda$ is negative for an attractive potential. Thus, if $l$ is odd, the Born term has the sign of the potential, $\lambda$, and approaches infinity. On the other hand, if $l$ is even, the sign is reversed. In the gap, the $S$ matrix is $S_{l}=1-2 f_{l}\left[-\nu /\left(\nu+M^{2}\right)\right]^{\frac{1}{2}}$. The two possibilities are shown in Fig. 1. These curves, of course, could cross the axis several times. It is obvious in any case that the function $S_{l}$ must have at least one zero in the gap for every other $l$. If there is a bound state present then $S$ is not bounded in the gap. The Born term discussion is not changed and since the residue of a bound-state pole in $S$ must be positive, the two possibilities are as shown in Fig. 2.

To summarize the situation with an example, we consider the $l=0$ partial wave for the case of an attractive potential. We have seen that if there is no bound state, then $S_{0}$ must have at least one zero in the gap. If the potential is made attractive enough to produce a bound state, the zeroes may disappear from the gap. We return later to an instance of this sort.

The discussion of a theory in which there is no one-particle exchange, or Born term (for example, $\pi-\pi$ scattering), is less conclusive. One trivial statement which can be made is that if the scattering amplitude is positive near the negative cut, which starts at $\nu=-\mu^{2}, S_{l}$ must have a zero. The infinity in this case comes from the phase-space factor $\rho$.

FIG. 2. $S$ matrix with a bound state.

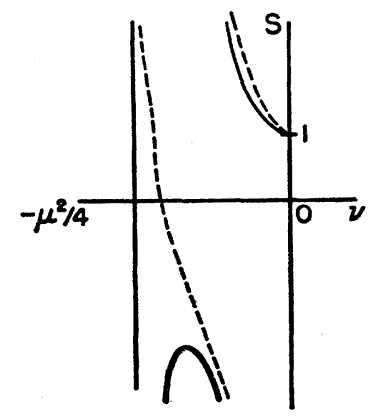


Let us turn to a discussion of the nature of the cuts of a partial wave amplitude. Consider the general problem of the type of singularity at $s=a$ of the function

$$
J^{\mathrm{I}}(s)=\frac{1}{\pi} \int_{a}^{\infty} d s^{\prime} \frac{W\left(s^{\prime}\right)}{s^{\prime}-s} .
$$

On the unphysical Riemann sheets, defined by crossing the cut in the neighborhood of $a$, we have, for example,

$$
J^{\mathrm{II}}(s+i \epsilon)=J^{\mathrm{I}}(s+i \epsilon)-2 i W^{\mathrm{I}}(s+i \epsilon),
$$

and

$$
J^{\mathrm{III}}(s+i \epsilon)=J^{\mathrm{I}}(s+i \epsilon)-2 i\left[W^{\mathrm{I}}(s+i \epsilon)+W^{\mathrm{II}}(s+i \epsilon)\right]
$$

where, if the point $a$ is a branch point of $W$, we choose the cut to run towards $+\infty$. Thus if $W^{\mathrm{I}}(s)$ has a square-root type of singularity at $s=a$, then $J^{\mathrm{III}}=J^{\mathrm{I}}$ and $J$ also has a square-root type of branch cut. However, if $W$ is analytic in the neighborhood of $s=a$, then $J$ has a logarithmic singularity.

The general form of the partial wave amplitude for the process depicted in Fig. 3, i.e., $2 M_{a} \rightarrow 2 M_{b}$ through a state of $2 \mu$ 's, is

$$
G(s)=\frac{1}{\pi} \int_{-\infty}^{a} d s^{\prime} \frac{g\left(s^{\prime}\right)}{s^{\prime}-s}+\frac{1}{\pi} \int_{4 \mu^{2}}^{\infty} d s^{\prime} \frac{B\left(s^{\prime}\right)}{s^{\prime}-s^{\prime}},
$$

where

$$
B(s)=\rho(s) H_{a}(s) H_{b}(s)\left[1-2 i_{\rho}(s) f_{\pi-\pi}(s)\right]^{-1},
$$

and $H_{[a, b]}$ is the partial wave annihilation amplitude for the process $2 M_{[a, b]} \rightarrow 2 \mu$.

Since $B(s)$ has a square-root singularity at $s=4 \mu^{2}$ coming from the explicit factor of $\rho(s)$, the positive branch cut in $G(s)$ connects only two Riemann sheets. It is clear that this two-sheetedness property holds for each two-particle singularity in a multichannel situation.

If there is a one-particle exchange diagram, then its contribution to $g(s)$ has singularities of the form $\left[\left(s-4 M_{a}^{2}\right)\left(s-4 M_{b}^{2}\right)\right]^{-\frac{1}{2}}$. Thus $g(s)$ is analytic in the neighborhood of $s=a$ and the left-hand cut of $G$ is logarithmic in nature.

The discussion in the cases that have no one-particle exchange graph is more involved. Let us consider for definiteness the case of pion-pion scattering. If the complications due to isotopic spin are neglected, the results of Chew and Mandelstam are that $a=0$, and

$$
\begin{aligned}
g_{l}(s)=\frac{2}{\nu} \int_{0}^{-\left(\nu+\mu^{2}\right)} d \nu^{\prime} & P_{l}\left(1+2 \frac{\nu^{\prime}+\mu^{2}}{\nu}\right) \\
& {\left[\sum_{l^{\prime}=0}^{\infty} \alpha_{l^{\prime}} P_{l^{\prime}}\left(1+2 \frac{\nu+\mu^{2}}{\nu^{\prime}}\right) \operatorname{Im} G_{l^{\prime}}\left(\nu^{\prime}\right)\right], }
\end{aligned}
$$

where $\nu=\left(s-4 \mu^{2}\right) / 4$. Since we have just shown that $\operatorname{Im} G_{l^{\prime}}\left(\nu^{\prime}\right)$ has a square-root singularity at $\nu^{\prime}=0$, this implies $g_{l}(s)$ has a square-root type of singularity at

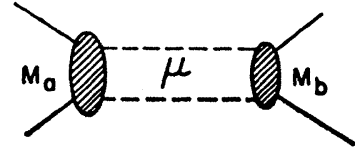

FIG. 3. Scattering graph.

$s=0$. In fact, it is easily seen that

$$
g_{l}(s) \simeq P_{l}(-1) a_{0}^{2}(-s)^{\frac{3}{2}}
$$

where $a_{0}$ is the $S$-wave scattering length. In addition, $g_{l}(s)$ has a logarithmic cut starting at $s=4 \mu^{2}$. The square-root behavior at $s=0$ implies that the negative cut in $G_{l}(s)$ from two-particle exchange connects two Riemann sheets. This result is true in more general circumstances. For example, in nucleon-nucleon scattering, the two-pion exchange contribution is easily shown to be two-sheeted in character.

Let us now turn to a discussion of the scattering amplitude without expanding in partial waves.

\section{FIXED ANGLE}

In order to discuss the analyticity of the scattering amplitude at a fixed angle, we assume that a Mandelstam representation holds in the physical sheet. This then allows a determination of the radius of convergence of a partial wave expansion on the second sheet.

The scattering amplitude $F$ is written in the form

$$
\begin{aligned}
F^{\mathrm{I}}(\nu, z)=\int_{\mu^{2}}^{\infty} d u^{\prime} & A_{2}\left(u^{\prime}, \nu\right) /\left[u^{\prime}+2 \nu(1+z)\right] \\
& +\int_{\mu^{2}}^{\infty} d t^{\prime} A_{3}\left(t^{\prime}, \nu\right) /\left[t^{\prime}+2 \nu(1-z)\right]
\end{aligned}
$$

Subtractions do not affect our general conclusions and are therefore suppressed. The only property of the weight functions $A_{2}$ and $A_{3}$ that is needed is that they are analytic functions of $\nu$ with a cut along the positive real axis.

$$
\operatorname{Im} F^{\mathrm{I}}(\nu, z)=\rho(\nu) \int \frac{d \Omega^{\prime}}{4 \pi} F^{\mathrm{I}}(\nu+i \epsilon, x) F^{\mathrm{I}}\left(\nu-i \epsilon, z^{\prime}\right),
$$

where

$$
x=z z^{\prime}+\left[\left(1-z^{2}\right)\left(1-z^{\prime 2}\right)\right]^{\frac{1}{2}} \cos \phi^{\prime} .
$$

In exact analogy with the partial-wave discussion, the scattering amplitude on the second sheet is introduced as

$$
F^{\mathrm{II}}(\nu, z)=F^{\mathrm{I}}(\nu, z)-2 i \rho(\nu) \int \frac{d \Omega^{\prime}}{4 \pi} F^{\mathrm{I}}(\nu, x) F^{\mathrm{II}}\left(\nu, z^{\prime}\right) .
$$

It is convenient to transform this into a nonsingular integral equation of the form

$$
F^{\mathrm{II}}(\nu, z)=F^{\mathrm{I}}(\nu, z)-2 i \int_{-1}^{1} d z^{\prime} K\left(z, z^{\prime} ; \nu\right) F^{\mathrm{II}}\left(\nu, z^{\prime}\right),
$$

where

$$
K\left(z, z^{\prime} ; \nu\right)=\frac{\rho(\nu)}{4 \pi} \int d \phi^{\prime} F^{\mathrm{I}}(\nu, x) .
$$


This azimuthal integration is carried out readily and the result is

$$
\begin{aligned}
K\left(z, z^{\prime} ; \nu\right) & =\frac{\rho(\nu)}{4 \pi} \int d u^{\prime} A_{2}\left(u^{\prime}, \nu\right)\left[\left(1+z z^{\prime}+u^{\prime} / 2 \nu\right)^{2}\right. \\
& \left.-\left(1-z^{2}\right)\left(1-z^{\prime 2}\right)\right]^{-\frac{1}{2}}+\frac{\rho(\nu)}{4 \pi} \int d t^{\prime} A_{3}\left(t^{\prime}, \nu\right) \\
& \times\left[\left(1-z z^{\prime}+t^{\prime} / 2 \nu\right)^{2}-\left(1-z^{2}\right)\left(1-z^{\prime 2}\right)\right]^{-\frac{1}{2}} .
\end{aligned}
$$

Our next task is to solve the integral Eq. (3.3) for $F^{\mathrm{II}}(\nu, z)$ and to discuss its analyticity in $\nu$ and $z$. Even if $F^{\mathrm{I}}$ did not satisfy a Mandelstam representation, it is clear from the integral equation that the domain of analyticity of $F^{\mathrm{II}}$ is closely connected with that of $F^{\mathrm{I}}$, except for poles arising from the homogeneous equation and the trivial kinematical cut from the explicit $\rho(\nu)$ factor. The Fredholm solution to this equation can be examined readily. This solution can be written in the canonical form

$$
F^{\mathrm{II}}(\nu, z)=F^{\mathrm{I}}(\nu, z)+\frac{1}{D(\nu)} \int_{-1}^{1} d z^{\prime} N\left(z, z^{\prime} ; \nu\right) F^{\mathrm{I}}\left(\nu, z^{\prime}\right),
$$

where $N$ and $D$ are the usual Fredholm determinants.

Since the region of integration is finite, it follows from standard arguments that the analyticity domain of $F^{\mathrm{II}}(\nu, z)$ in $\nu$ is at least as large as that of $F^{\mathrm{I}}\left(\nu, z^{\prime}\right)$ for all $z^{\prime}$, except that there are the cuts from $\rho(\nu)$ and the possibility of zeroes of $D(\nu)$.

The connection between these poles and the ones discussed earlier in the partial wave amplitudes is made apparent by considering the eigenfunctions of the kernel. If we write

$$
K\left(z, z^{\prime} ; \nu\right)=\sum(2 l+1) P_{l}(z) P_{l}\left(z^{\prime}\right) \rho(\nu) f_{l}(\nu),
$$

then it follows from general arguments ${ }^{7}$ that

$$
D(\nu)=\prod_{l=0}^{\infty}\left[1+2 i \rho(\nu) f_{l}(\nu)\right] .
$$

From these results it is possible to discuss the analyticity in $Z$ for fixed complex $\nu$. In particular, we are interested in the possibility of making a partialwave expansion of $F^{\mathrm{II}}(\nu, z)$. If the nearest singularities in $z$ are either complex or real but a finite distance outside the interval $(-1,1)$, it is possible to pass an ellipse inside these points enclosing the physical region. Then an expansion in a Legendre series is convergent within this region.

From the expression for the kernal $K\left(z, z^{\prime} ; \nu\right)$, it is readily seen that for fixed $\nu$ there is analyticity in $z$ except when

where

$$
z^{2}+z^{2}+(1+x / 2 \nu)^{2} \pm 2 z z^{\prime}(1+x / 2 \nu)=1
$$

$$
\mu^{2}<x<\infty, \quad-1<z^{\prime}<1 .
$$

${ }^{7}$ See, for example, W. V. Lovitt, Linear Integral Equations (McGraw-Hill Book Company, Inc., New York, 1924).
The condition that this singularity lie in the physical region of $z$ is $\nu \leqslant-\mu^{2} / 4$. Thus, as long as $\nu$ is not on the negative cut, a Legendre expansion is valid for $F^{\mathrm{II}}(\nu, z)$. Of course, if $\nu$ is in the neighborhood of a point where one of the $S_{l}(\nu)$ has a zero, say at $\nu_{l}$, then a singular term in the expansion, of the $\Gamma_{l} P_{l}(z) /\left(\nu-\nu_{l}\right)$, is present.

\section{FIXED MOMENTUM TRANSFER}

We take up now the analytic properties of the scattering amplitude on the second sheet as a function of the energy at fixed momentum transfer. We content ourselves with a brief discussion, since a more complete treatment has been given by Zimmerman. ${ }^{8}$ The integral equation for the second sheet amplitude is evidently

$F^{\mathrm{II}}\left(\nu, t_{13}\right)=F^{\mathrm{I}}\left(\nu, t_{13}\right)$

$$
-2 i \rho(\nu) \int d \Omega_{2} F^{\mathrm{I}}\left(\nu, t_{12}\right) F^{\mathrm{II}}\left(\nu, t_{23}\right),
$$

where the subscripts on the momentum transfer mean

$$
t_{i j}=-2 \nu\left(1-z_{i j}\right) .
$$

Now assuming that a two-dimensional representation holds on the physical sheet, the azimuthal integration can be carried out as in the previous section. One obtains the integral equation given previously, Eq. (3.4), with the understanding that $z$ is to be expressed in terms of $\nu$ and $t_{13}$. Since for fixed $z_{2}, F^{\mathrm{II}}\left(\nu, z_{2}\right)$ is analytic in the cut plane, this integral equation representation implies that $F^{\mathrm{II}}(\nu, t)$ is analytic in a region bounded by the vanishing of one of the denominators:

where

$$
\left(\lambda^{\prime} \lambda \pm z_{2}\right)^{2}-\left(\lambda^{\prime 2}-1\right)\left(\lambda^{2}-1\right)=0,
$$

$$
\lambda^{\prime}=1+t^{\prime} / 2 \nu, \quad \lambda=1+t / 2 \nu .
$$

Solving for $\nu$, we find

$$
\nu=-\left\{t+t^{\prime} \pm\left[2 t t^{\prime}\left(1 \pm z_{2}\right)\right]^{\frac{1}{2}}\right\} / 2\left(1 \mp z_{2}\right) .
$$

Since in the physical region, $t<0$, and also $t^{\prime}>0$, these roots are complex;

where

$$
\nu=x \pm i y,
$$

$$
x=-\left(t+t^{\prime}\right) / 2\left(1 \mp z_{2}\right) ; \quad y^{2}=-t t^{\prime}\left(1 \pm z_{2}\right) / 2\left(1 \mp z_{2}\right)^{2} .
$$

For fixed $t^{\prime}$ this point moves on a branch of a hyperbola as $z_{2}$ varies. Eliminating $z_{2}$, we get

$$
y^{2}=-t t^{\prime} x\left(4 x+t+t^{\prime}\right) /\left(t+t^{\prime}\right)^{2} .
$$

The boundary of the region of analyticity occurs when $t^{\prime}$ reaches its minimum value $\mu^{2}$. The equation of the boundary curve is

$$
y^{2}=-t \mu^{2} x\left(4 x+\mu^{2}+t\right) /\left(t+\mu^{2}\right)^{2} .
$$

If $t>-\mu^{2}$, then the left-hand branch of this hyperbola is the boundary, whereas if $t<-\mu^{2}$, the right-hand

${ }^{8} \mathrm{~W}$. Zimmerman (private communication). 
branch takes over. This analyticity region is, of course, not the largest possible since we have not used any analytic properties of the kernels in the integral equation. Thus we consider the iterated solution to see if a larger region emerges.

Using our previous results, the solution can be written as

where

$$
F^{\mathrm{II}}(\nu, t)=F^{\mathrm{I}}(\nu, t)+\sum_{N} I_{N}
$$

$$
\begin{gathered}
I_{N}=(-2 \pi i \rho)^{N} \int d t_{0} \cdots d t_{N} d z_{0} \cdots d z_{N-1} w\left(\nu, t_{0} \cdots t_{N}\right) \\
\times\left[\left(\lambda_{N} \lambda-z_{N-1}\right)^{2}-\left(\lambda_{N}^{2}-1\right)\left(\lambda^{2}-1\right)\right]^{-\frac{1}{2}} \cdots \\
\quad \times\left[\left(\lambda_{1}^{2} z_{1}-z_{0}\right)^{2}-\left(\lambda_{1}^{2}-1\right)\left(z_{1}^{2}-1\right)\right]^{-\frac{1}{2}}\left(\lambda_{0}-z_{0}\right)^{-1},
\end{gathered}
$$

and

$$
\lambda_{i}=1+t_{i} / 2 \nu .
$$

The weight function $w$ is an analytic function of $\nu$ in the plane cut along the positive real axis. Any permutation of the $\lambda$ 's in the integrand is permissible, since it becomes a symmetric function after the $z_{i}$ integrations are performed.

The integration over $z_{0}$ can be done immediately and the result can be studied as a function of $z_{1}$; for $\nu$ in the physical region, the integral over $z_{0}$ can be written in the form

$$
\int_{z_{1}{ }^{\prime}(\nu)}^{\infty} d \lambda_{1}{ }^{\prime}\left[\chi\left(\lambda_{0}, \lambda_{1}, \lambda_{1}{ }^{\prime}\right)\right]^{-1}\left(\lambda_{1}{ }^{\prime}-z_{1}\right)^{-1},
$$

where

and

$$
z_{1}^{\prime}(\nu)=\lambda_{0} \lambda_{1}+\left[\left(\lambda_{0}^{2}-1\right)\left(\lambda_{1}^{2}-1\right)\right]^{\frac{1}{2}}
$$

$$
\chi(x, y, z)=\left[x^{2}+y^{2}+z^{2}-2 x y z-1\right]^{\frac{1}{2}} .
$$

Now introduce the variable $\eta_{1}$ given by

$$
\lambda_{1}{ }^{\prime}=\lambda_{0} \lambda_{1}+\eta_{1}\left[\left(\lambda_{0}{ }^{2}-1\right)\left(\lambda_{1}{ }^{2}-1\right)\right]^{\frac{1}{2}} .
$$

The integral becomes

$$
\int_{1}^{\infty} d \eta_{1}\left(\eta_{1}^{2}-1\right)^{-\frac{1}{2}}\left(\lambda_{1}^{\prime}-z_{1}\right)^{-1}
$$

Now the integral over $z_{1}$ can be performed and the above argument repeated successively. The final representation for $I_{N}$ that we consider is

$$
\begin{aligned}
I_{N}=(-2 \pi i \rho)^{N} \int d t_{0} & \cdots d t_{N} w\left(\nu, t_{0} \cdots t_{N}\right) \\
& \times \int_{1}^{\infty} \frac{d \eta_{N}}{\left(\eta_{N}{ }^{2}-1\right)^{\frac{1}{2}}} \cdots \frac{d \eta_{1}}{\left(\eta_{1}^{2}-1\right)^{\frac{1}{2}}} \frac{1}{\lambda_{N}{ }^{\prime}-z},
\end{aligned}
$$

where

$$
\lambda_{i}{ }^{\prime}=\lambda_{i} \lambda_{i-1}{ }^{\prime}+\eta_{i}\left[\left(\lambda_{i}{ }^{2}-1\right)\left(\lambda_{i-1}{ }^{2}-1\right)\right]^{\frac{1}{2}} .
$$

This function has branch points in the $\nu$ plane for $\lambda_{i}{ }^{2}=1$, or $\nu=-t_{i} / 4$ and $\nu=\infty$, where $I_{N}$ becomes logarithmically divergent. In addition, there is a branch point when

$$
\lambda_{N}{ }^{\prime}-z=0,
$$

with $\eta_{i}=1$ for all $i$. This equation is symmetric in all the $\lambda$ 's and may be written

where

$$
z=\cosh \left(\xi_{0}+\xi_{1}+\cdots \xi_{N}\right)=1+t / 2 \nu,
$$

$$
\cosh \xi_{i}=\lambda_{i}
$$

The roots of this equation are, in general, complex. Therefore, one concludes that for a fixed momentum transfer, $F^{\mathrm{II}}$ has complex singularities.

\section{APPLICATIONS}

The simplest applications of these results seem to be in discussions involving individual partial waves. We are interested in those processes in which the singularities on the second sheet should be quite important. The most obvious example is one in which the singularities on the physical sheet conspire in such a manner as to force $S_{l}$ to have a zero in the gap just below the physical cut. The resulting pole on the second sheet has a dramatic effect on low-energy scattering. This can easily occur even if there are no nearby singularities on the physical sheet. A nearby pole on the second sheet can be just as important as a bona fide boundstate pole. A second example is found in the problem of the anomalous thresholds. In this case, we know that one is forced to extend the physical cut into the gap region. Thus, if one of these poles were present in the integrand, it might cause a breakdown of the dispersion representation' which could not be discerned from perturbation theory. We now turn to a detailed discussion of these problems.

\section{A. Anomalous Thresholds}

As a first application of the previous results, consider the form factor for a scalar particle of mass $M_{a}$ which interacts with a scalar photon through a pair of scalar particles of mass $\mu$ as illustrated in Fig. 4. For this discussion it proves illuminating to follow the procedure developed by Mandelstam ${ }^{1}$ instead of the equivalent method described by Blankenbecler and Nambu, ${ }^{2}$ since in the former method, the difficulty with poles on the second sheet seem superficially more dangerous. The latter authors introduce a representation of the form factor which has only normal cuts and then they continue to the physical sheet. The Mandelstam procedure makes use of the analyticity of the Green's functions in the masses and continues from the normal to the anomalous case. In both of these methods one is forced to continue certain functions to their second Riemann sheet and it is this aspect of the problem which is of interest here.

Following Frazer and Fulco, ${ }^{9}$ the form factor in the normal case $\left(M_{a}\right.$ small) can be written in the form

${ }^{9}$ W. R. Frazer and J. R. Fulco, Phys. Rev. 117, 1609 (1960). 
FIG. 4. Form factor graph.

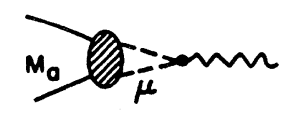

$$
F(s)=\frac{1}{\pi} \int_{4 \mu^{2}}^{\infty} d s^{\prime}\left[A\left(s^{\prime}\right)\right] /\left[s^{\prime}-s\right]
$$

where

$$
\begin{aligned}
A(s)=\rho(s) \exp [ & \left.\Delta^{*}(s)+\Delta(s)\right] \\
& \times \int_{-\infty}^{a} d t(t-s)^{-1} \alpha(t) \exp [-\Delta(t)] .
\end{aligned}
$$

Here $\alpha(t)$ is the discontinuity across the negative cut in the partial wave amplitude for the annihilation process and $\Delta(s)$ is the usual line integral over the relevant phase shift of $\mu-\mu$ scattering, $\mathcal{J}_{4 \mu^{2}}{ }^{\infty} d s^{\prime} \delta\left(s^{\prime}\right) /$ $\left(s^{\prime}-s\right)$.

If an analytic continuation to larger values of the external mass $M_{a}$ is made by giving it a small negative imaginary part, the point $a$ moves in the path illustrated by the solid line in Fig. 5. The line integral from $4 \mu^{2}$ to infinity in $F(s)$ must be deformed to avoid this protruding branch cut of $A(s)$ as indicated by the dotted line. In order to perform these continuations, one must introduce the $S$ matrix in the form

$$
\exp \left[\Delta^{*}(s)\right]=\exp [\Delta(s)] S^{-1}(s)
$$

and also continue the factor $\exp [-\Delta(t)]$ in the integrand of $A$ onto its second sheet as the upper limit $a$ moves around the point $4 \mu^{2}$. These continuations yield

$$
\begin{aligned}
A(s)= & \rho(s) \exp [2 \Delta(s)] S^{-1}(s) \\
& \times\left\{\int_{-\infty-i \eta}^{4 \mu^{2}-i \eta} d t(t-s)^{-1} \alpha(t) \exp [-\Delta(t)]\right. \\
& \left.-\int_{4 \mu^{2}+i \eta}^{a+i \eta} d t(t-s)^{-1} \alpha(t) S(t) \exp [-\Delta(t)]\right\},
\end{aligned}
$$

where we have used the fact that the function $\alpha(t)$ has a square-root type cut starting at $4 \mu^{2}$.

One might superficially expect that when the anomalous threshold $a$ reaches the point where $S_{i}$ has a zero, the continuation would break down in a manner foreign to perturbation theory. This is not the case, since from the integral over $\alpha(t)$ a factor of $S$ appears to cancel any such pole. The final result after collapsing the line integral to the real axis is ${ }^{10,11}$

Fig. 5. Anomalous threshold behavior.

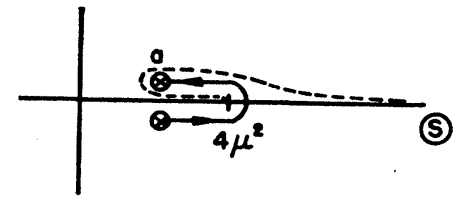

10 This result was also obtained by $\mathrm{R}$. Oehme (to be published). ${ }^{11}$ For an application of this procedure to the vector magnetic moment of the sigma particle, see R. Marr, L. Landovitz, and R. Blankenbecler, Bull. Am. Phys. Soc. 6, 80 (1961) (also, to be published).

$$
\begin{array}{rl}
F(s)=\frac{1}{\pi} \int_{a}^{4 \mu^{2}} d s^{\prime}\left(s^{\prime}-s\right)^{-1} & B\left(s^{\prime}\right) \\
& +\frac{1}{\pi} \int_{4 \mu^{2}}^{\infty} d s^{\prime}\left(s^{\prime}-s\right)^{-1} C\left(s^{\prime}\right),
\end{array}
$$

where

and

$$
B(s)=2 \pi i \rho(s) \alpha(s) \exp [\Delta(s)]
$$

$$
\begin{array}{r}
C(s)=\rho(s) \exp \left[\Delta^{*}+\Delta\right]\left\{\int_{-\infty}^{a} d t(t-s)^{-1} \alpha(t) \exp [-\Delta(t)]\right. \\
\left.+\int_{a}^{4 \mu^{2}} d t(t-s)^{-1} \alpha(t) \exp [-\Delta(t)][1+S(t)]\right\} .
\end{array}
$$

Results similar to the form factor case hold also for the anomalous scattering situation depicted in Fig. 3. The absorptive part of the scattering matrix $G$ has the form

$$
\begin{aligned}
& \qquad \operatorname{Im} G(s)=\rho(s) H_{b}^{*}(s) H_{a}(s), \\
& \text { where } \\
& \begin{aligned}
& H_{[a, b]}(s)=\exp [\Delta(s)] \int_{-\infty}^{[a, b]} d t(t-s)^{-1} \\
& \times \exp [-\Delta(t)][\alpha(t), \beta(t)],
\end{aligned}
\end{aligned}
$$

if $M_{a}$ and $M_{b}$ are sufficiently small. First consider the case where only $M_{a}$ is large enough for an anomalous threshold. By the same procedure as before, the scattering amplitude $G(s)$ is found to be

$$
G(s)=\frac{1}{\pi} \int_{a}^{\infty} d l(l-s)^{-1} J(t)
$$

where the imaginary part of $G$ in the anomalous region, $a<s<4 \mu^{2}$, is

$$
J(s)=2 \pi i \rho(s) \alpha(s) H_{b}(s) .
$$

Now if the mass $M_{b}$ is increased until $b>a$, which can obviously occur even if $H_{b}(s)$ has a normal threshold, then $J$ becomes complex. The condition $b>a$ is just the condition found in perturbation theory by Karplus, Sommerfield, and Wichmann" for the "super" anomalous case. There is no ambiguity or difficulty in continuing past this point if all the masses are given negative imaginary parts as required by the definition of the Green's function of interest. The essential point here for our purposes is again that the superficially dangerous factor of $S^{-1}$ cancels. It would seem that in any approximate evaluation of a scattering amplitude with anomalous thresholds, one must make sure that the approximations made do not destroy this cancellation.

\section{B. Bound States}

Another interesting application of the analytic properties of the scattering amplitude on the second sheet

${ }^{12}$ R. Karplus, C. M. Sommerfield, and E. H. Wichmann, Phys. Rev. 111, 1187 (1958). 


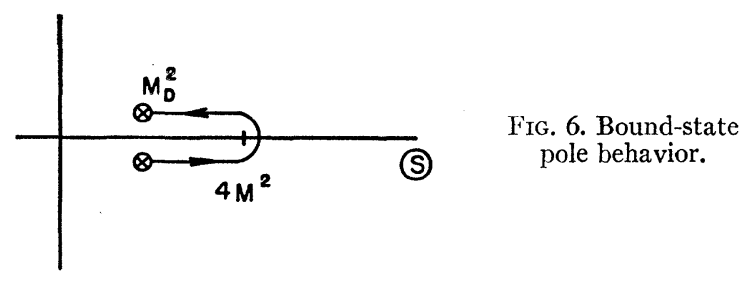

is found in the problem of bound states. One may entertain the question of whether any calculational program based on unitarity and analyticity is complete in the sense that it yields the masses and coupling constants of bound states in terms of more fundamental constants. The difficulty is that the bound state must be present in the sum over states in the unitarity condition. This seems to introduce arbitrary constants. We show that by making very reasonable assumptions about the analyticity of the production amplitudes, the bound-state problem can be solved completely within such a framework.

A physical example is found in nucleon-nucleon scattering. If one applies the standard $N / D$ procedure without explicitly putting in the deuteron pole, then it is reasonable to expect $D$ to develop a zero at the deuteron mass. This can even be demonstrated rigorously in the case of potential scattering. However, in field theory a new problem arises. The entire mass spectrum singularities due to the deuteron must be generated. For example, the contribution to the inelastic physical cut from the $n+p+\pi$ intermediate state must extend its threshold from $(2 M+\mu)^{2}$ to $\left(M_{d}+\mu\right)^{2}$, and this extra cut must have the twosheetedness properties associated with two-particle cuts. The canonical explanation is that these extra poles and cuts migrate from the second sheet. We show in detail why this explanation is correct.

We consider scalar nucleon-nucleon scattering as an example. The process nucleon-nucleon scattering is called reaction one with energy $s$. The crossed processes, nucleon-antinucleon scattering, have energies $t$ and $u$. The Mandelstam representation is written in the form

$$
G(s, t, u)=\frac{1}{\pi} \int_{4 M^{2}}^{\infty} d s^{\prime}\left(s^{\prime}-s\right)^{-1} \operatorname{Im} f\left(s^{\prime}\right)+G^{\prime}(s, t, u),
$$

where $f(s)$ is the $l=0$ partial wave amplitude. Therefore

$$
\operatorname{Im} f(s)=\rho(s) f(s) f(s) S^{-1}(s),
$$

in the elastic region.

The essential point is to recall now that if there are no bound states and the effective potential is attractive, $S$ has a zero for $s$ in the gap. As the potential strength increases, this zero is expected to move towards the physical region, $s>4 M^{2}$. Guided by what does occur in potential scattering, we assume that this zero moves in the path illustrated in Fig. 6 and that the scattering amplitude is an analytic function of the position of this zero. We need not assume analyticity in the coupling constant. This zero is trapped in the gap and on the real axis. Either it moves in the path shown or it never reaches $4 M^{2}$. In the latter case the analyticity of the amplitude does not change. When the zero passes around the point $4 M^{2}$, the line integral over $\operatorname{Im} f(s)$ must be analytically deformed to avoid this wandering pole. The deformed path can be shrunk to a small circle about the pole plus the contribution from $4 M^{2}$ to infinity. The small circle yields a contribution to $G$ of the form

$$
\Gamma /\left(s-M_{d}^{2}\right),
$$

where the pole has been placed at $M_{d}{ }^{2}$. This is the mechanism by which poles move from the second to the first sheet of the scattering amplitude as true bound states are formed.

Now we examine the contribution of the $n+p+\pi^{0}$ inelastic intermediate state before a bound state has formed. The absorptive part of $G$ probably cannot be expanded in the relative angular momentum of the two nucleons. However, we restrict our attention to the configuration where the neutron and proton are in an $l=0$ state without making such an expansion. This particular contribution to the absorptive part of $G$ therefore can be written as an integration over the center-of-mass energy of the nucleon pair and the angle variables of the pion. The result, except for constant factors, is

$$
\begin{aligned}
A^{\prime}=\int d \Omega & \int_{2 M}^{s^{\frac{1}{3}}-\mu} d W M^{*}\left(s, W, \Omega_{f}, \Omega\right) M\left(s, W, \Omega, \Omega_{i}\right) \\
& \times\left(W^{2}-4 M^{2}\right)^{\frac{1}{2}}\left[\frac{\left(W^{2}-s-\mu^{2}\right)^{2}}{4 s}-\mu^{2}\right]^{\frac{1}{2}}
\end{aligned}
$$

The general form for the production amplitude $M$ must now be discussed. Since the neutron and proton are in a relative $S$ state, perturbation-theoretic arguments suggest that we can write

$$
M\left(s, W, \Omega, \Omega_{i}\right)=J\left(s, W, \Omega, \Omega_{i}\right) \exp \left[\Delta\left(W^{2}\right)\right]
$$

where $\Delta\left(W^{2}\right)$ is the line integral over the neutron-proton $S$-wave phase shift, and $J$ is a very complicated complex function which we cannot completely characterize at the moment but it does not have the physical cut in $W^{2}$. We assume that whatever its properties, they do not interfere with the following discussion.

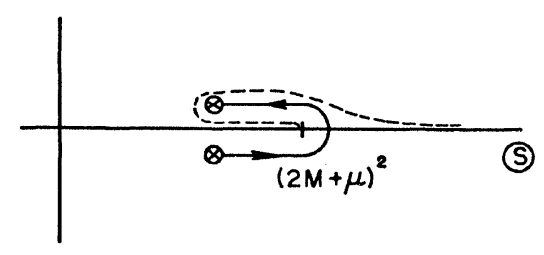

FIG. 7. Inelastic cut behavior as a bound state develops. 
When the amplitude $A^{\prime}$ is formed, there occurs a factor of $\exp \left[\Delta^{*}\right]$, which must be rewritten as $\exp [\Delta] S^{-1}\left(W^{2}\right)$. Now we further restrict our attention to the pole term in $S^{-1}$, which occurs at $M_{d}{ }^{2}$. The other singularities need not be discussed further. Then the pole contribution to the absorptive part can be written as

$$
\begin{gathered}
A^{\prime \prime}(s)=\int_{2 M}^{s^{\frac{1}{2}-\mu}} d W\left(W^{2}-4 M^{2}\right)^{\frac{1}{2}}\left[\left(W^{2}-s-\mu^{2}\right)^{2}-4 s \mu^{2}\right]^{\frac{1}{2}} \\
\times\left(W^{2}-M_{d^{2}}\right)^{-1} \int \frac{d \Omega}{2 s^{\frac{1}{2}}} J^{*}\left(s, W, \Omega_{f}, \Omega\right) \\
\quad \times J\left(s, W, \Omega, \Omega_{i}\right) \exp \left[2 \Delta\left(W^{2}\right)\right] .
\end{gathered}
$$

We now need to discuss the analyticity of $A^{\prime \prime}$ as a function of $s$. The singularity which is of interest to us is one of the endpoint singularities due to the pole at $M_{d}{ }^{2}$. These occur at $\left(s^{\frac{1}{2}}-\mu\right)^{2}=M_{d}{ }^{2}$. The branch point closest to the physical cut is $s^{\frac{1}{2}}=\mu+M_{d}$. If the coupling is now increased, this branch point moves in a path shown as the solid line in Fig. 7 (see also Fig. 6). Then the line integral over $A^{\prime \prime}$ from $(2 M+\mu)^{2}$ to infinity must be deformed to the dotted line in Fig. 7 to avoid this oncoming branch cut. When this deformed integral is collapsed to the real axis, it can be rewritten as a line integral from $\left(M_{d}+\mu\right)^{2}$ to infinity. Thus the correct two particle cut has been generated in the same manner as an anomalous threshold.

Similar statements hold for all the higher inelastic states. Thus, if our assumptions about the structure of production amplitudes are true, a new particle of mass $M_{d}$ has been added to the mass spectrum.

This argument concerning bound states can also be used to clarify the problem of unstable particles. First, assume that $S_{l}$ has a complex zero at $M^{* 2}$, which is near the physical cut and produces a scattering resonance. We have seen that if such a pole exists on the second sheet across the elastic cut, then there is a branch cut starting at $s=\left(M^{*}+\mu\right)^{2}$ on the unphysical sheet across the three-particle branch line. This cut can be drawn parallel to the real axis toward plus infinity, if we like. One possible interpretation which is consistent with the identification of $M^{* 2}$ as a pole due to a one (unstable) particle state is that this latter branch line singularity represents the rescattering of a pion with the unstable particle in the intermediate state. If there is a resonance in the three-particle system $(n+p+\pi)$, then it should show up in the $s$ dependence of the function $J$. One would like $J^{*}$ to have a simple pole in order to be consistent with the interpretation in the two-particle case. It still is not clear that this is a consistent and/or unique interpretation of these types of singularities.

\title{
Excitation Spectrum in Many-Boson Systems*
}

\author{
Fuminiko Takano $\dagger$ \\ Metcalf Chemical Laboratories, Brown University, Providence, Rhode Island
}

(Received March 14, 1961)

\begin{abstract}
When only two-body correlations are fully taken into account, there appears an energy gap in the excitation spectrum for many-boson systems as shown by Girardeau and Arnowitt and confirmed by Wentzel. This energy gap is shown to disappear and the spectrum to become phononlike again and proportional to the momentum for small momentum, if we construct the eigenmodes of excitations (collective excitations), taking into consideration appropriate higher-order terms.
\end{abstract}

\section{INTRODUCTION}

$\mathbf{M}$ ANY authors have studied the many-boson system and showed the presence of the phonon-roton type excitation spectrum as in the actual system of liquid helium. Especially, Bogoliubov ${ }^{1}$ noticed the fact that the occupation number of the zero-momentum state was macroscopically large, and treated the quantum amplitude for this state $a_{0}^{\dagger}, a_{0}$ as a classical number $N_{0}^{\frac{1}{2}}$. He could get, then, the phononlike spectrum by diagonalizing the quadratic terms of

* Supported in part by the Alfred P. Sloan Foundation.

$\dagger$ On leave of absence from Tokyo University of Education, Tokyo, Japan.

${ }^{1}$ N. N. Bogoliubov, J. Phys. (U.S.S.R.) 9, 23 (1947). $a_{\mathrm{k}}^{\dagger}, a_{\mathbf{k}}(|\mathbf{k}| \neq 0)$ in the Hamiltonian. Brueckner and Sawada $^{2}$ used essentially the same method and confirmed this result.

Recently, Girardeau and Arnowitt ${ }^{3}$ showed that there appeared an energy gap in the excitation spectrum if one used the best trial function which fully took into consideration two-body correlations. This result was confirmed by Wentzel ${ }^{4}$ who used a slightly different method.

Though they obtained a better ground-state energy

${ }^{2}$ K. A. Brueckner and K. Sawada, Phys. Rev. 106, 1117, 1128 (1957).

${ }^{3}$ M. Girardeau and R. Arnowitt, Phys. Rev. 113, 755 (1959).

${ }^{4}$ G. Wentzel, Phys. Rev. 120, 1572 (1960). 